\title{
A DAM journey in a mature business
}

\author{
Gregory Crandall \\ is a content and solutions consultant with responsibility for management of web services, technology and processes to support digital \\ asset management needs at Whirlpool Corporation.
}

Keywords: mature business, enterprise solution, user defined categories, tiger team, enterprise architecture

\begin{abstract}
Whirlpool Corporation deployed digital asset management through several implementations. The initial thrust was developing skill sets and an understanding of the available technology within its Creative Works group. This led to an implementation that allowed creative production staff to organize digital assets and assure the ability to locate those assets for repurposing. As technology advanced new tools became available that allowed additional functionality and more robust management of assets. This included support of Lightweight Directory Access Protocol (LDAP) protocols to offer single sign-on, asset-level security to manage assets throughout the lifecycle of the products, and a self-serve environment that allowed creating web services to meet varying needs of a diverse user group. These groups consisted of super users among the creative staff, agencies and marketing department, as well as the more general users of the corporate environment and of our trade partners.
\end{abstract}

\section{A DAM JOURNEY IN A MATURE BUSINESS}

Whirlpool Corporation, the world's largest manufacturer of home appliances, began the journey of digital asset management (DAM) in the mid-nineties within its creative organization by recognizing the need for organizing, storing and easily retrieving digital assets for its own production requirements. The initial purpose for the first generation DAM system led the way to have available access to high resolution images for print production, sales catalogs and brochures for internal creative staff, and was the principal low-hanging fruit that would be the easiest to deploy and provide the quickest return on investment.

As the discovery and testing of solutions started, we found most solutions were based on a client-server environment. This lent to sharing information and use of the technology within a workgroup or centralized area with a heavy emphasis on the Macintosh client and its overwhelming use within the creative community. This was exactly the environment we were working in, and it became apparent that change management or acceptance would not be a high barrier to success. However, as we moved from the workgroup and Macintosh environment and started pursuing an enterprise solution or, at least, a solution readily available to our external agencies, it became more an issue of people and processes rather than of technology. With middleware maturing and web services developing, possibilities began to emerge allowing technology and these processes for managing digital assets to become available to the enterprise and beyond. With this, our next step was recognizing solutions that would meet technical governance issues related to the enterprise. These were stability, security and scalability, and to allow ease of use when deploying across the enterprise.

With technological advancement and skill sets developing new techniques and processes, benchmarking and the use of consultants started playing a leading role in determining best practices and available solutions. Ideas covered in benchmarking and consulting were determining how to begin supporting multiple channels beyond the traditional print environment and what technical architecture requirements were required to support those new practices. In addition, new technical developments emerged related to digital capture, allowing the creative/ photographic functions to directly create and capture in digital format. This eliminated the 
need for film and scanning, reducing costs and time in the creative process, allowing imagery to go directly to color management, and retouching and ingestion.

Through several iterations of our DAM solutions, providing user-defined categories and taxonomies started playing a critical role. Our approach was to define personas for differing user groups and, how they would expect to conduct searches.

This was only partially successful, but it did force an element of discipline in the ingestion process. Keeping in mind the end user, and how they might search or need imagery became a mantra in the production process. We also found that there is no easy answer to this question and it remains to be seen how the mix between predefined categories and free text searching meets the ultimate users' needs.

In an attempt to answer this, groups of users were engaged to assist in the creation of taxonomies and to provide feedback on how the system assisted them in their work. A formalized user group (tiger team) along with periodic surveys allowed us to receive direct feedback on the efficiencies of how we cataloged assets.

Certain defined categories were used for creating an environment that would provide guidance for users in predetermined searches and expected results. Here we provided drop-down menus to select from to pre-fill search forms, such as brands, product categories, and product colors.

The ingestion process is governed to assure consistency of keywords and subjective descriptions of imagery by having the role of media cataloger responsible for ingesting new assets. This role consists of assigning not only the keywords from the drop-down tables previously discussed, but also assigning consistent keywords to help describe the image environment, as well as other metadata determined by the content and how it is associated with the data models.

A further part of the ingestion process is the formal review of all new assets ingested.

Reviewing all new assets ingested for the prior week is carried out on a weekly basis. This allows an opportunity for proofing and verifying metadata associated with these new assets. Even though these practices are resourceintensive and require devotion of time, the time investment assures that the new asset is valid, searchable and retrievable. Based on the costs of creating the new content and its inherent value to the enterprise, this activity has proven to be of great value in providing the asset to the enterprise.

As part of our project management services, all projects begin with a meeting review between the marketing department and the appropriate agency managing the project. During this review, a template-driven spreadsheet is delivered outlining all the necessary product and image descriptions containing all of our required metadata fields with the actual attributes completed and reviewed. The benefit is that it allows a review of the project and assures that if new fields or attributes are required, sufficient time is allowed for changes to be made to the taxonomy before the approved image is ready for ingestion and distribution.

We often find that the best way to catalog an image is to have a peer review and conduct informal questioning from creative and production staff on relevant descriptions. This is critical when we move from factual-based images of stand-alone single product images to environmental images that support brand or lifestyle messages, or non product related images such as environmental, food or people (see Figure 1).

We also use contextual processing on our database, which extracts textual content and indexes the content as fully searchable words and phrases. This becomes useful when the content being ingested is PDF files derived from sales literature, catalogs or logos.

In addition to the requirements for meeting enterprise architecture standards, adoption and use of common taxonomies and data models is important. This underlying standard opened the opportunity for interoperability and synchronization of data within other enterprise applications and allows the introduction to the enterprise user with common expressions and ability to search for digital content. This enterprise user is familiar with common lexicons of product-related information and the ability to offer searches based on this factual-based data becomes more relevant in today's business environment.

Many of these metadata fields were specific to data models that were necessary to distinguish 


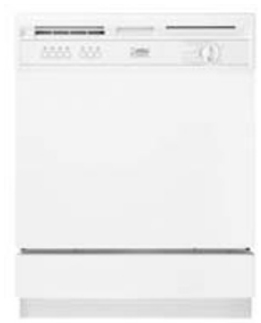

\begin{tabular}{l} 
Stand-alone product image \\
\hline Factual \\
Sku \\
Color \\
Brand
\end{tabular}

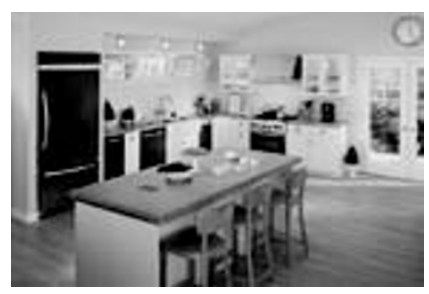

Product environment

Factual + Emotion

Multiple skus

Color

Brand

Brand message support

Ambience of environment

Lifestyle

Prop elements

Design elements

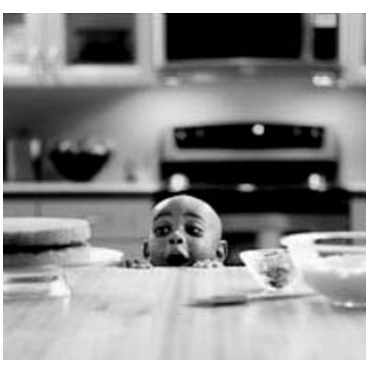

Environment - Non product Emotion

Gender

Prop description

Emotional description

Ethnicity

Figure 1: Image types

one type of content from another and also to distinguish and make easier for specific types of searches or needs. Based on these models, some attributes were global and used across all data models and content, such as brand; others were specific to content such, as usage guidelines for logos or library number, rights/music and rights/ talent for videos.

In addition, potential future integration opportunities were looked at when taxonomies were created, and certain fields in some of the data models were identified for possible future use, such as market sub headings, Uniform Product Code (UPC) code and material status code derived from SAP enterprise software.

Another requirement was the ability to generate reports from user logs that would assist in communicating ongoing value provided by our DAM solution. Minimally, we wanted to be able to observe activities based on searching and downloading activities. In addition to the costavoidance element of repurposing in general, download activities show a specific cost avoidance in regards to staffing and other personnel resources.

Our history showed that analog delivery activity on behalf of customers cost Whirlpool Corporation a minimum of $\$ 10$ for every file delivered, whether by $\mathrm{CD}$ or other means. Figure 2 represents YTD activity for 2004. On an overall basis, search activity exceeded 105,000 with downloading of digital files exceeding 33,000. Prior to our DAM implementation, historical activity of distributing files via other methods averaged 4,000 files per year. Not only were we showing increased usage of digital assets, we were substantially reducing costs for this activity as well.

Prior to February 2004, the availability of online searching for digital assets and manual setup of new user accounts was normally communicated through various channels. This was followed by individual training via telephone support or emailed PowerPoint

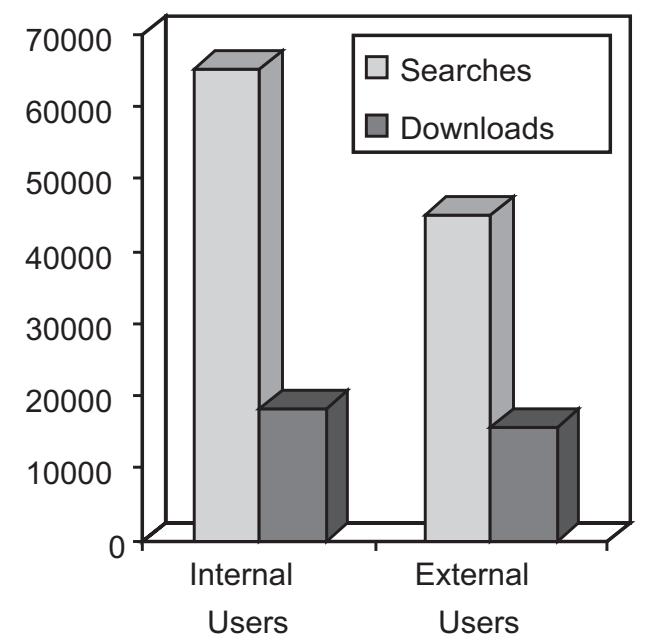

Figure 2: YTD activity for 2004 


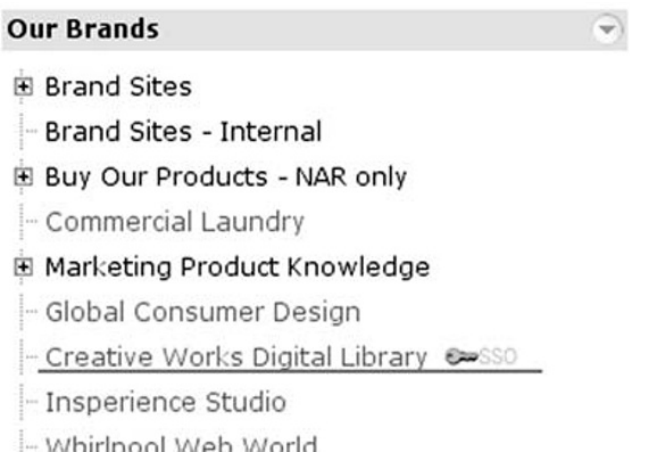

Figure 3: Corporate portal SSO

presentations. To promote an embedded use and to ease support requirements, an effort was made to create a single sign-on from various access points. The first was through a link on a portal supporting trade activities for promotions. Next, an Single Sign On protocol (SSO) link was created on the corporate portal (see Figure 3).

Since the rollout of the corporate portal SSO, activity by new users has increased by an annualized rate of 40 per cent and activity by internal users has now bypassed that of external users. In addition, this activity represents a tenfold increase in activity over historical levels prior to DAM introduction. In other words, yearly activity would previously range in the area of around 4-5,000 files, distributed via CD or other methods; now we are seeing this as a monthly average. This supports one of our main business drivers - brand consistency - and helps reinforce the idea that regardless of the channel or audience, a consistent product image is used. By the adoption of corporate standards for color consistency and accuracy we now see that branded colors and features are represented accurately with no additional costs across the entire marketing communications demand chain. A secondary benefit is that ad hoc requests from corporate users to our creative functions are lessened by creating automatic transformers from high resolution cyan-magenta-yellowblack (CMYK) images to red-green-blue (RGB) images for web publishing and presentation use and to support use of images for editorial use.

Real-time image processing plays a role in not only supporting our site's diverse user community, but it also provides a tool by reducing or eliminating hands-on work to images for specific channels. Corporate users can take advantage of a JPEG transformer that allows images to be easily placed into PowerPoint presentations without any work required on the image. In addition, multiple transformers have been created to allow agencies and production staff to download CMYK images to specific literature template sizes with imbedded International Color Consortium (ICC) profiles for accurate print output. Transformers have also been created to output images to specific web templates that support trade and internal websites with no additional PhotoShop work required.

As the current system was being developed, security, permissions, roles and organizations became necessary to the success of supporting product lifecycles and managing assets that had limited or restricted usage rights. Security is managed at the asset level, with roles and organizations being applied to access specific assets and certain functions within our site.

CMYK transformers are limited to creative, production and agency staff and restricted to corporate users who don't have the appropriate native applications to support certain file formats or color spaces.

In addition, with the recent deployment of streaming video assets, a business requirement was to manage access to video assets based on network facilities and bandwidth constraints. With our platform based on Java2 Enterprise Edition (J2EE) standards, modifications to the UI allowed us to modify the application to easily manage new business requirements. In this case, video assets had to be managed based on network constraints at individual facilities. Modification to the application allowed us to enable streaming to the appropriate destination, and in the case of insufficient bandwidth the application could intervene with a preview and metadata, along with a message to the user for contact information to order the required video.

These security roles allow support of the product lifecycle. Imagery can now be restricted to specific organizations for pre-launch activity, then as the product is launched access can be promoted to various other organizations and ultimately to the entire user population. Likewise, as the process is reversed and we see the obsolescence of certain skus we can demote these related images until they become an audit asset with access only by administrators. 
With the progress to date on DAM, the next steps relate to business rules and logic and governance within the marketing communications demand chain. The current environment contains multiple silos with differing accountabilities and responsibilities, and even different goals, and the challenge will be to accommodate these needs.

Ultimately, we see DAM as a back-end application to manage rich media and provide utility to other enterprise applications. This would be via distributed content, based on business rules, and tailor-made content. In this scenario, as new content is created, logic can dictate formatting, naming, resolution, sizing and other specifics, published to those systems or users as they are needed. This will take asset repurposing to new levels. In today's environment, we know that a new image will be used on average 12 times during its lifecycle. In the new scenario, we envision an asset being used hundreds of times. In this new era we could distribute the image automatically for internal websites, trade partners' sites and other systems including our enterprise resource planning (ERP) and customer relationship management (CRM) systems. This, in turn, could deliver to the individual consumer in a pre-buy situation an image for their review, or possibly in a customized personal letter or brochure.

The next step is to identify our optimized processes and create an environment that would allow rapid adoption of changes to the business. Of course, the underlying issues that will continue to offer challenges are people and processes. Technology will always be able to provide the basic solution. However, it is our people and processes that can ignite reinvention, and that in itself will be the test in taking our mature company into a new realm of digital asset management. 